\title{
A Software Framework for Creating Patient Specific Geometric Models from Medical Imaging Data for Simulation Based Medical Planning of Vascular Surgery
}

\author{
Nathan Wilson ${ }^{1}$, Kenneth Wang ${ }^{2}$, Robert W. Dutton ${ }^{3}$, Charles Taylor ${ }^{4}$ \\ ${ }^{1}$ CISX 305, Stanford University, Stanford, CA, 94305-4075 \\ nwilson@stanford.edu \\ ${ }^{2}$ CISX 332, Stanford University, Stanford, CA, 94305-4075 \\ wang@gloworm.stanford.edu \\ ${ }^{3}$ CISX 333, Stanford University, Stanford, CA, 94305-4075 \\ dutton@gloworm.stanford.edu \\ ${ }^{4}$ Division of Biomechanical Engineering, Durand 213, \\ Stanford University, Stanford, CA 94305-3030 \\ taylorca@stanford.edu
}

\begin{abstract}
The primary purpose of vascular surgery is to restore blood flow to organs and tissues. Current methods of vascular treatment planning rely solely on diagnostic and empirical data to guide the decision-making process. This paper details a simulation-based medical planning system for cardiovascular disease that use computational methods to evaluate alternative surgical options prior to treatment using patient-specific models of the vascular system. A software framework, called Geodesic, is introduced that reduces the time required to build patient-specific models from medical imaging data from several weeks to less than one day.
\end{abstract}

\section{Introduction}

The current paradigm for surgery planning for the treatment of cardiovascular disease relies exclusively on diagnostic imaging data to define the present state of the patient, empirical data to evaluate the efficacy of prior treatments for similar patients, and the judgment of the surgeon to decide on a preferred treatment. This paper details a simulation-based medical planning system for cardiovascular disease that uses computational methods to evaluate alternative surgical options prior to treatment using patient-specific models of the vascular system. The blood flow (hemodynamic) simulations enable a surgeon to see the flow features resulting from a proposed operation and to determine if they pose potential adverse effects such as increased risk of atherosclerosis and thrombosis formation. A significant bottleneck for simulationbased medical planning, however, is the lengthy time required to build patient specific geometric models and associated difficulties in discretization and numerical simulation. This paper details a software framework, called Geodesic, that reduces the time required to build patient-specific geometric models from medical imaging data from several weeks to less than one day. Geodesic includes an integrated level set kernel 
for image segmentation of blood vessel (lumen) boundaries and prepares the appropriate input files for hemodynamic simulation.

Cardiovascular surgeons currently rely on medical imaging data and past experience to form an operative plan. Taylor et. al. [1] proposed a new paradigm in vascular surgery, namely that computer simulation could be used to provide a surgeon with quantitative and qualitative comparisons of different operative plans prior to surgery to augment the information already available. This requires a software system that can be used by medical technicians in conjunction with vascular surgeons to perform numerical simulations predicting the blood flow for several different operative procedures.

\section{Simulation Based Medical Planning}

The first system developed for simulation based medical planning (SBMP) of vascular surgery was the "Stanford Virtual Vascular Laboratory" [2]. This system predominantly relied on idealized models of the vascular system. Specifically, circular crosssections were used to describe the surface of each artery branch to be included in the model and these individual artery models were joined (boolean union) together to create a solid model representing the vascular system. This solid model could then be automatically meshed with unstructured tetrahedral elements using an automatic mesh generator. Finally, input files were automatically created for a finite element fluid mechanics solver to perform hemodynamic simulation of the given vascular system.

A major advantage of using idealized models was the ability to use parametric models, which enabled studies of a wide variety of vascular anatomy to be performed in a short period of time. The major shortcoming of the SVVL was its inability to build patient specific preoperative geometric models from medical imaging data. A second generation system for vascular surgery planning (named ASPIRE) is detailed in [2]. This system had an internet-based user interface specifically designed for a vascular surgeon. However, like the SVVL this system also lacked the ability to build 3-D patient-specific geometric models from medical imaging data.

This paper will detail recent advances in the development of a software system, named Geodesic, which is a modular framework that greatly simplifies the process of building preoperative vascular models from medical imaging data and performing subsequent postoperative planning. The Geodesic framework consists of several key modules including: a level set module for segmentation of imaging data, a solid modeling module for geometric computation, a meshing layer for querying meshes for information required to specify boundary conditions and creating input files for hemodynamic solvers, and an interactive graphical user interface (GUI) for easily creating alternative post operative plans. 


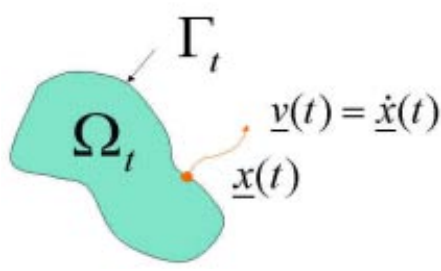

(a)

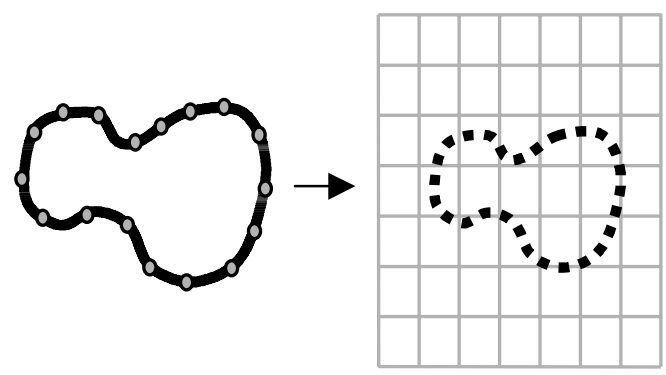

(b) (c)

Fig.1: The level set function. Figure (a) shows the abstract mathematical representation, while (b) shows 1-D boundary edges being embedded in a 2-D uniformly spaced grid (c).

\section{Image Segmentation Using Level Set Methods}

Numerous methods exist for segmentation of boundaries from medical imaging data. For example, thresholding is a technique that defines boundary edges as a given value of image intensity. A contouring algorithm, such as marching cubes, is then used to extract the iso-surface yielding the boundary. A more powerful and increasingly popular approach, introduced by Osher and Sethian [3], is known as the level set method. Briefly, the level set method imbeds a boundary of interest in a higher dimension space, and solves a partial differential equation governing the evolution of the interface (see Fig. 1). That is, we define a scalar function $\phi$ such that:

$$
\phi(\underline{x}(t), t)=0 \quad \underline{x} \in \Gamma
$$

Using the chain rule and taking the time derivation of (1), the definition of the normal from differential geometry, and projecting the velocity function along the normal to the boundary, transforms the expression of (1) into the standard partial differential equation of the level set method:

$$
\begin{array}{lll}
\phi_{t}+\underline{v}(t) \cdot \nabla \phi=0 \quad & \Rightarrow & \phi_{t}+F|\nabla \phi|=0 \\
\text { where } \underline{n}=\frac{\nabla \phi}{|\nabla \phi|} & \text { and } \quad \underline{v}=F \underline{n}
\end{array}
$$

Choosing $\phi$ as the signed distance function (i.e. $|\nabla \phi|=1$ ) and making the velocity normal to the curve depend on boundary curvature and the gradient of the image data 
intensity (i.e. $\mathrm{F}=\mathrm{F}(\underline{\mathrm{x}}, \mathrm{t}, \kappa,|\nabla I|)$ ) completes the problem specification. Standard finite-difference numerical methods for the solution of Hamilton-Jacobi equations are employed to evolve a surface and extract the vessel boundary. A two stage process is employed similar to that described in [4], where the curve is initially evolved "close" to the boundary and then a second iteration is used to center the extracted curve in the band of high intensity gradient near the edge of the boundary. Fig. 2 shows an example segmentation of a vessel lumen from MRI data. A detailed discussion of the level set method for vascular surgery applications can be found in [5].

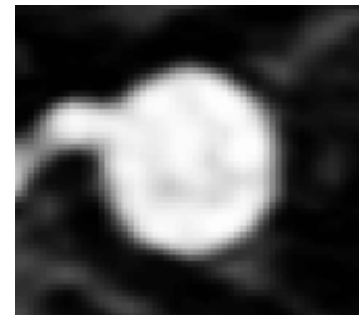

(a)

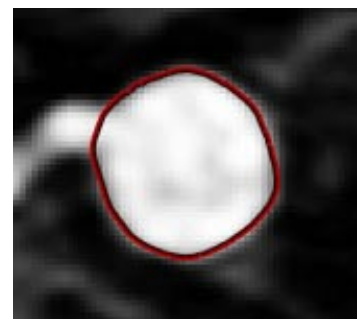

(b)

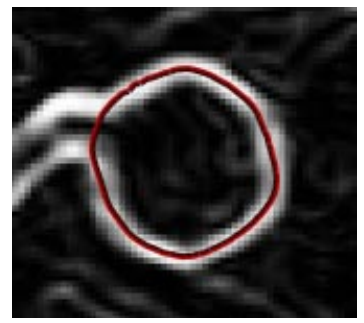

(c)

Fig. 2: Level set segmentation of a vessel boundary. (a) shows the original MR image slice, (b) shows the final boundary on the image data, and (c) shows the final boundary on a plot of the magnitude of the gradient of the image intensity (where white represents a larger absolute value of the gradient). This example shows the need for curvature feedback when evolving the surface, that is the curvature dependence prevents the boundary from "leaking" into the smaller adjacent branch vessel.

\section{Preoperative Patient-Specific Model Construction}

The level set method discussed previously can be used in any number of spatial dimensions, and Geodesic has an integrated multi-dimensional level set kernel. While using the 3-D level set method theoretically has many advantages such as the ability to directly extract volumetric geometry from image data, it has several significant practical disadvantages. First, the method imbeds the evolving boundary in a higher dimension space (i.e. a surface defining a volume represented using triangular facets requires a 3-dimensional grid). Thus memory and computational cost scale roughly by $\mathrm{O}\left(\mathrm{n}^{3}\right)$. Additionally, the variability in the image data can make the constants found in the boundary velocity functions used to do the segmentation a function of position. Finally, the human body contains a vast network of arteries, but it may be desired to simulate only a subset of them. It is difficult to extract an accurate geometric representation using the level set method in 3-D while preventing the front from advancing into undesired branches. 
For these reasons, the technique used in the current work is to extract 2-D cross sections of the lumen boundary along the path of a given arterial branch, and then use solid modeling operations to "loft" an analytic (NURBS) surface skinning the surface of the contours. This process can be seen in Fig. 3. The process is repeated for each desired branch in the image data. In more detail, the first step is for the user to define a path for a given branch. This can be done in two ways, with the user selecting multiple points defining the path or automatically using a custom program to extract the path given an initial and final point. Once the path is obtained, level set simulations are performed on selected cross sections along the path. Typically, a subset of these cross-sections are chosen by the user to create a lofted solid model of the given branch.

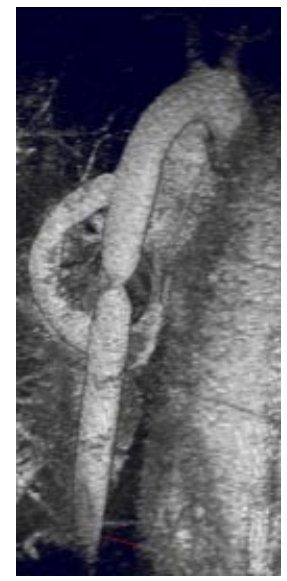

(a)

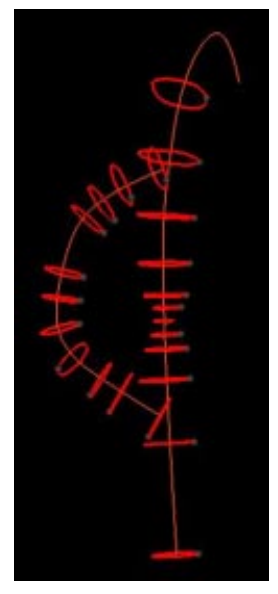

(b)

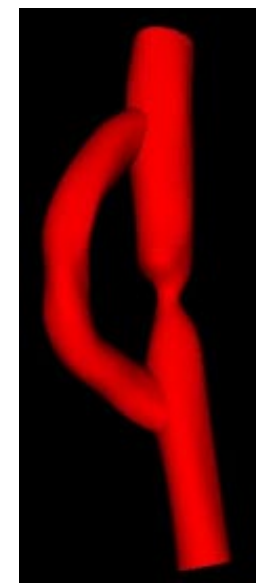

(c)

Fig. 3: Solid model construction of a bypass model for a pig with an artificially created stenonsis. (a) shows the image data, (b) shows the paths along which selected level set curves have been extracted, and (c) shows the resulting solid model after the joining of the bypass and aorta branches.

When desired branches have been created, they are joined (i.e. boolean union) together in the solid modeler to create a single solid representing the vascular system. This solid model is then meshed using automatic mesh generation methods [6], and hemodynamic solver input files are automatically created (see Fig. 5). Note that an additional advantage of building the system from a set of branches is the information needed to assign boundary conditions for simulation is implicitly specified during the creation of the resulting solid. That is, the "cap" of each branch is a planar cross section tagged with an integer id and the branch name (the tagged parameters are called "attributes"). The solid modeler being used propagates the attributes during boolean operations, so when the final solid is being created all of the outflow boundaries are automatically marked. This is because for all branches, except possibly the aorta, one of the two caps will be absorbed (i.e. eliminated) in the final solid. The remaining 
named surface is then assumed to be the outflow boundary. This eliminates the user from needing to interact with the mesh to select element faces or nodes for boundary conditions, which is key to going automatically from patient geometric models to simulation results.

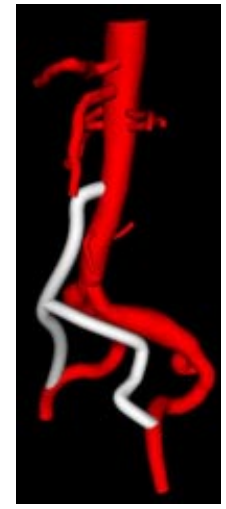

(a)

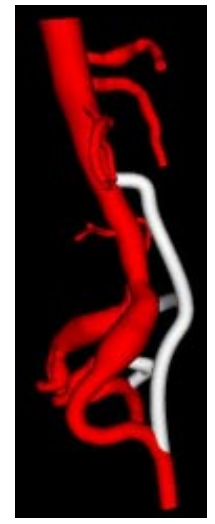

(b)

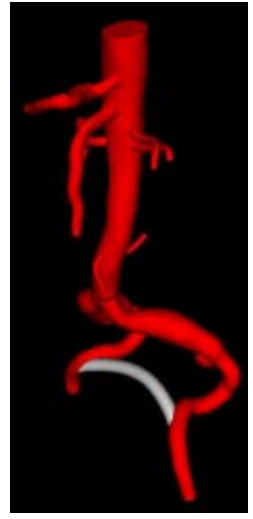

(c)

Fig. 4: Two different operative plans for a human patient. (a) and (b) show two different views of a bifurcating bypass, while (c) shows a femoral-femoral bypass. These bypasses were created with slices of the image data in the same rendering window to assist in making anatomically correct (i.e. physically possible) bypasses.

\section{Creating Alternative Operative Plan Models}

The ability to evaluate different treatment options and predict the impact of different procedures on a given patient is of critical importance in patient specific vascular surgery planning. Additionally, these procedures should be planned in the presence of the imaging data so that the validity of the proposed procedure is insured. Fig. 4 shows several different images of potential treatment options for an individual patient. The operative planning GUI inside of Geodesic is simple, yet powerful. Slide bars control the location of three image slices $(\mathrm{x}, \mathrm{y}, \mathrm{z})$ oriented in the image volume. In addition, a cursor is controlled by slide bars and positioned in 3-space. The user selects desired points by moving the cursor, and the path is highlighted in the rendering window as the user selects points. This allows the user to verify that the proposed operation is physically possible given the patient anatomy. Currently this operation requires significantly less time than the preoperative model construction, as three or four clinically relevant operation alternatives can be created in less than one hour. 


\section{Mesh Generation and Simulation}

As a first approximation, it can be assumed that the vessel walls do not deform (i.e. are rigid) and that blood can be approximated as a Newtonian fluid with constant material parameters. In addition, it is assumed that the outlet pressure of each of the branches is known and can be set to a constant pressure (i.e. $p=0$ ). With these assumptions, the hemodynamic simulation reduces to solving the Navier-Stokes equations of fluid mechanics with periodic inflow boundary conditions. Two types of boundary conditions have been explored. First, as an approximation the analytic inflow profile (which assumes fully developed, axisymmetric flow) proposed by Womersley [7] can be prescribed on the inflow boundary. The only required input for the analytic profile is the volumetric flow rate as a function of time. Second, the inlet velocity can be directly determined from physiologic data using phase-contrast MRI.

Fig. 5 shows a mesh of a pig that underwent an aortic bypass, as well as a simulation of blood flow in the system. This model was created completely inside of Geodesic and is currently being compared with in vivo experimental results.

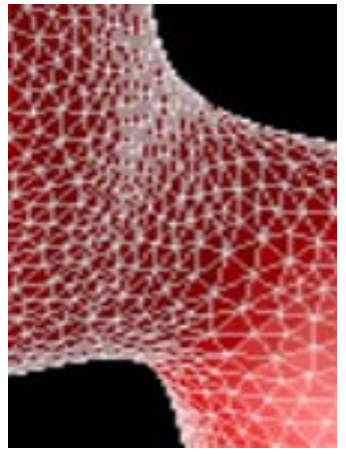

(a)

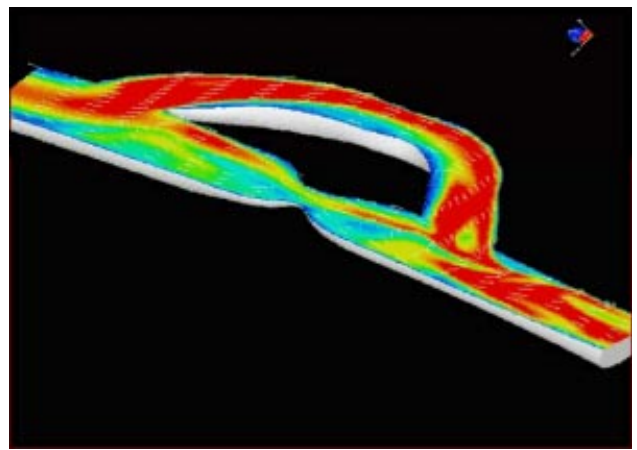

(b)

Fig.5: Mesh and simulation results of a pig aortic-bypass. (a) shows a close up of the surface mesh near the stenonosis, while (b) shows the magnitude of the flow velocity (blue low, red high) in the aorta and bypass.

\section{Conclusions and Future Work}

A software framework, called Geodesic, was introduced that reduces the time required to build patient-specific models from medical imaging data from several weeks to less than one day. Further improvements in the model construction, mesh generation and flow solution processes are essential for these simulation-based medical planning methods to be clinically feasible. In addition, work still remains in automating the 
specification of physiologic inflow boundary condition data from PC-MRI. In vivo studies comparing animal (pig) experiments and the simulation results to validate the assumptions being used are currently underway.

\section{References}

1. Taylor, C. A.: A Computational Framework for Investigating Hemodynamic Factors in Vascular Adaptation and Disease. Phd thesis, Stanford University (1996)

2. Taylor, C.A., Draney, M.T., Ku, J.P., Parker, D., Steele, B.N., Wang, K., Zarins, C.K.: Predictive Medicine: Computational Techniques in Therapeutic Decision-Making. Computer Aided Surgery (1999) 4:231-247

3. Osher, S., Sethian, J.A.: Fronts propagating with curvature-dependent speed: Algorithms based on Hamilton-Jacobi formulations. J. Computational Physics (1988) 79:12-49

4. Malladi, R., Sethian, J.A.: A Real-Time Algorithm for Medical Shape Recovery. Proc. Intl. Conf. Computer Vision (1998) 304-310

5. Wang, K.: Level Set Methods for Computational Prototyping with Application to Hemodynamic Modeling. Phd thesis, Stanford University (expected 2001)

6. Shephard, M.S., Georges, M.K.: Automatic three-dimensional mesh generation by the finite octree technique. Int. J. Numerical Methods in Eng. (1991) 32:709-749

7. Womersley, J.R.: Method for the calculation of velocity, rate of flow and viscous drag in arteries when the pressure gradient is known. J. Physiology (1955) 53:502-514 